\title{
STEM in 4 Dimensions: Using Multivariate Analysis of Ptychographic Data to Reveal Material Functionality
}

Stephen Jesse ${ }^{1,2}$, Miaofang Chi ${ }^{1,3}$, Albina Y. Borisevich ${ }^{1,3}$, Alex Belianinov ${ }^{1,2}$, Raymond R. Unocic ${ }^{1,2}$, Christopher T. Symons ${ }^{1,5}$, Eirik Endeve ${ }^{1,4}$, Richard K. Archibald ${ }^{1,4}$, Sergei V. Kalinin ${ }^{1,2}$, Andrew R. Lupini $^{1,3}$

${ }^{1}$ Institute for Functional Imaging of Materials, Oak Ridge National Laboratory, Oak Ridge, TN 37831

${ }^{2}$ Center for Nanophase Materials Sciences, Oak Ridge National Laboratory, Oak Ridge, TN 37831

${ }^{3}$ Materials Science \& Technology Division, Oak Ridge National Laboratory, Oak Ridge, TN 37831

${ }^{4}$ Computer Science and Mathematics Division, Oak Ridge National Laboratory, Oak Ridge, TN 37831

${ }^{5}$ Computational Sciences \& Engineering Division, Oak Ridge National Laboratory, Oak Ridge, TN 37831

The scanning transmission electron microscopy (STEM) is a powerful platform for studying materials and their many varied properties (including structural, electronic, magnetic, ferroic etc.) locally and at their fundamental length-scales. In a general sense, STEM imaging can be represented as an information channel connecting the interactions of a highly focused electron beam and sample to the observer. However, in the current standard implementation, this channel is severely restricted by the instrumental linkage in which the information rich electron diffraction pattern (Ronchigram) is reduced to a single value (e.g. through the integrated intensity over a relatively large detector) at each beam location thus resulting in loss and distortion of information about important but subtle aspects of material properties. Previous theoretical and experimental work [1,2,3,4] suggests that full acquisition of the Ronchigram at each spatial location during a scan can enable super resolution, phase-contrast imaging, imaging of internal fields, and 3D sample reconstruction. Data acquisition and storage has evolved to a point now that it is possible to capture high resolution 4D ptychography data sets rapidly, and the use of large-scale compute facilities enables the processing and mining of these multi-GB data sets to distil the most salient aspects of the data while separating the statistically significant variations (signal) from noise.

In the current work we have utilized the DE-12 camera (Direct Electron, LP, San Diego, CA), equipped with a 4096 x 3072 pixels Direct Detection Device (DDD®) sensor [5] installed on an aberration corrected FEI Titan operating at $300 \mathrm{kV}$ combined through a custom FPGA control system to synchronize frame capture and beam positioning to acquire 4D scanning-scattering data sets. Typical scans of 192X192 pixel images containing 384X384 Ronchigrams at every pixel were captured in less than 1 minute. A host of multivariate statistical methods can be brought to bear to extract meaningful information from these large data sets. Statistical methods can be advantageous in that they are parallelizable and can be efficiently implemented on large-scale computing platforms, are model-free and operate with no pre-imposed expectation or bias, and are capable of elucidating inter-pixel correlations unlikely to be noticed by human observation alone.

As an example, shown in Figure 1a is a micrograph rendered by integrating over the total intensity of Ronchigrams at each spatial location and shows the positions of atomic columns in thin film polymorph bismuth ferrite at a tetragonal/rhombohedral phase boundary. A combination of principal component analysis (PCA) and k-means clustering applied to the 4D data set enabled compression and sorting of the 16384 Ronchigrams into 25 spatially resolved groupings of similar scattering behavior. As shown in figure $1 \mathrm{~b}$, the different clusters are color-coded and the locations of atomic columns are indicated by an overlaid contour map. The most prominent result of this analysis is the clear differentiation of the two 
crystalline phases. Note this differentiation was based entirely on extracting subtle but statistically significant variations in Ronchigrams. Additionally, regular tiling of cluster arrangements commensurate with unit cell spacing provides a means to reveal, in a systematic way, the effects of local fields on electron scattering behavior. Figure 2 shows in greater detail the particular Ronchigrams associated with selected positions near an atomic column (indicated in figure 1b). Additional examples, more in-depth analysis methods, and relationships of analysis results to material properties will be discussed. [6]

\section{References:}

[1] J. M. Rodenburg, B. C. McCallum and P. D. Nellist, Ultramicroscopy, 48, (1993), p. 304.

[2] N. Shibata et al, Nature Physics, 8, (2012), p. 611.

[3] T.J. Pennycook et al, Ultramicroscopy, in press, (available on-line Nov. 2014)

[4] C. Ophus et al, Microsc. Microanal. 20, 2014

[5] The authors would like to acknowledge Liang Jin and Benjamin Bammes for their assistance in allowing us to use the DE-12 system. http://www.directelectron.com/.

[6] Research supported by: Oak Ridge National Laboratory's Center for Nanophase Materials Sciences (CNMS), which is sponsored by the Scientific User Facilities Division, Office of Basic Energy Sciences, U.S. Department of Energy, by the Laboratory Directed Research and Development Program of Oak Ridge National Laboratory, managed by UT-Battelle, LLC, for the U. S. Department of Energy, and by Division of Materials Sciences and Engineering Division, Office of Basic Energy Sciences, U.S. DOE. This study used the resources of the Oak Ridge Leadership Computing Facility at the Oak Ridge National Laboratory, and analysis under support of applied mathematics program at the DOE.
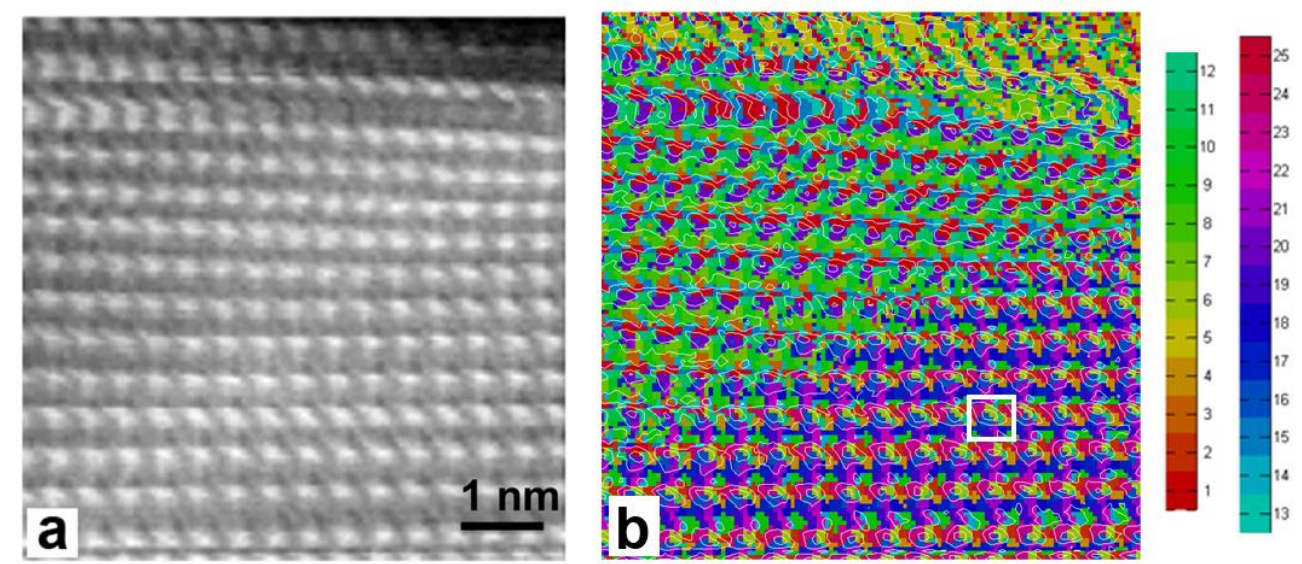

Figure 1. (a) Micrograph of tetragonal/rhombohedral phase boundary of thin film Bismuth Ferrite. (b) Ronchigram based cluster map clearly differentiating phases as well as local but regular variations in scattering behavior commensurate with unit cell tiling. Color bar indicates cluster labeling.
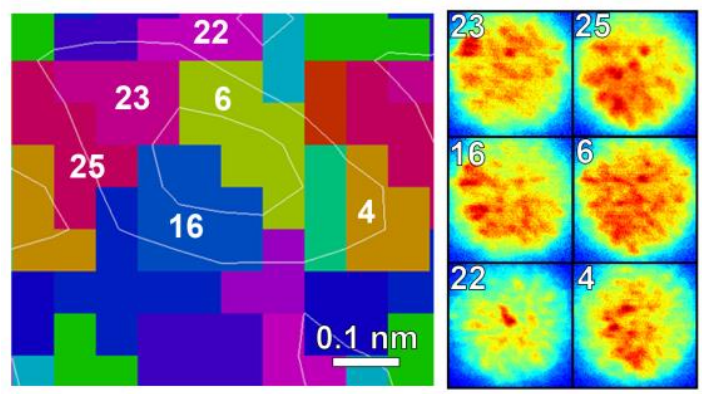

Figure 2. A zoomed-in view of the region indicated in figure $1 \mathrm{~b}$ illustrating the Ronchigrams associated with specific clusters in the vicinity of an atomic column. 